\title{
Interaction of the tetracyclines with double-stranded RNAs of random base sequence: new perspectives on the target and mechanism of action
}

\author{
Chinwe U Chukwudi ${ }^{1,2,3}$ and Liam Good ${ }^{1}$
}

The 16S rRNA binding mechanism proposed for the antibacterial action of the tetracyclines does not explain their mechanism of action against non-bacterial pathogens. In addition, several contradictory base pairs have been proposed as their binding sites on the 16S rRNA. This study investigated the binding of minocycline and doxycycline to short double-stranded RNAs (dsRNAs) of random base sequences. These tetracyclines caused a dose-dependent decrease in the fluorescence intensities of 6-carboxyfluorescein (FAM)-labelled dsRNA and ethidium bromide (EtBr)-stained dsRNA, indicating that both drugs bind to dsRNA of random base sequence in a manner that is competitive with the binding of $\mathrm{EtBr}$ and other nucleic acid ligands often used as stains. This effect was observable in the presence of $\mathrm{Mg}^{2+}$. The binding of the tetracyclines to dsRNA changed features of the fluorescence emission spectra of the drugs and the CD spectra of the RNA, and inhibited RNase III cleavage of the dsRNA. These results indicate that the double-stranded structures of RNAs may have a more important role in their interaction with the tetracyclines than the specific base pairs, which had hitherto been the subject of much investigation. Given the diverse functions of cellular RNAs, the binding of the tetracyclines to their double-stranded helixes may alter the normal processing and functioning of the various biological processes they regulate. This could help to explain the wide range of action of the tetracyclines against various pathogens and disease conditions

The Journal of Antibiotics (2016) 69, 622-630; doi:10.1038/ja.2015.145; published online 20 January 2016

\section{INTRODUCTION}

The tetracyclines are a group of broad-spectrum antibiotics that are known to act by inhibiting the binding of aminoacyl-tRNA to the mRNA-ribosome complex, thereby inhibiting protein synthesis., ${ }^{1,2}$ They are generally believed to bind to the $16 \mathrm{~S}$ ribosomal RNA, which is composed of a 1540 nucleotide RNA. In addition to their use as antibacterial agents, the tetracyclines are also known to be effective in the treatment of non-bacterial infections; for example, in the treatment of protozoan diseases such as giardiasis ${ }^{3}$ and viral diseases such as West Nile fever. ${ }^{4}$ They also possess anti-inflammatory, ${ }^{5,6}$ anti-apoptotic ${ }^{7}$ and neuroprotective properties. ${ }^{8}$ There is little indication of the mechanism(s) of action that underlie many of the reported activities. $5,9,10$ Because of the similarities between the bacterial ribosome and mitochondrial ribosome, it was believed that the anti-protozoal activities of the tetracyclines were mediated via a similar interaction with the mitochondrial ribosome of these parasites. ${ }^{11}$ However, the susceptibility of other protozoan species that lack mitochondria (for example, Trichomonas vaginalis, Giardia lamblia and Entamoeba histolytica) $)^{1,3}$ as well as viruses and helminths raises further questions about the exact target site(s) and molecular mechanism(s) of action of the tetracyclines.
Several studies have explored the binding of the tetracyclines to the small ribosomal subunit of different bacterial species with a view to identify the exact target site. ${ }^{12}$ A number of binding sites have been identified on the $16 \mathrm{~S}$ rRNA through photoaffinity labelling and chemical footprinting, indicating certain bases as contributing to the binding pocket. ${ }^{13-15}$ However, there have been varied and sometimes conflicting reports with regard to which bases within the $16 \mathrm{~S}$ rRNA form the core target site(s). ${ }^{14,16}$ It has been reported that tetracyclines induce stabilization of various cellular mRNAs in bacteria, indicating a more generalized interaction/effect on RNA. ${ }^{17}$ Interestingly, the viruses against which the tetracyclines have shown some efficacy are RNA viruses (for example, West Nile fever virus, ${ }^{7}$ Japanese encephalitis virus $^{18}$ and human immunodeficiency virus ${ }^{19-21}$ ). Bearing in mind that the $16 \mathrm{~S}$ ribosomal RNAs (like most cellular RNAs) fold to form double-stranded secondary structures which are potential sites for interaction with small molecules; $;^{22,23}$ and that the flat polycyclic structure and size of the tetracyclines confers potential for intercalation between base pairs of nucleic acids, the binding of the tetracyclines to double-stranded RNA (dsRNA) is worth considering as a possible target site on the $16 \mathrm{~S}$ rRNA (other than the proposed conflicting base pairs) that could allow the drugs bind to and act

${ }^{1}$ Department of Pathology and Infectious Diseases, Royal Veterinary College, University of London, London, UK and ${ }^{2}$ Department of Veterinary Pathology and Microbiology, University of Nigeria, Nsukka, Enugu State, Nigeria

${ }^{3}$ Current address: Department of Veterinary Pathology and Microbiology, University of Nigeria, Nsukka, Enugu State, Nigeria.

Correspondence: Dr C Chukwudi, Department of Veterinary Pathology and Microbiology, University of Nigeria, Nsukka Campus, Nsukka EN2 NSK1, Enugu State, Nigeria.

E-mail: chinwe.chukwudi@unn.edu.ng

Received 8 October 2015; revised 2 November 2015; accepted 8 December 2015; published online 20 January 2016 
against microbes that lack the $16 \mathrm{~S}$ ribosomal RNA (such as viruses and some protozoa). If such binding exists irrespective of the RNA base pairs (which is highly conserved in bacterial rRNA), then it could account for the wide range of action of the tetracyclines. In this study, the interactions of minocycline $\left(\mathrm{C}_{23} \mathrm{H}_{27} \mathrm{~N}_{3} \mathrm{O}_{7}\right)$ and doxycycline $\left(\mathrm{C}_{22} \mathrm{H}_{24} \mathrm{~N}_{2} \mathrm{O}_{8}\right)$ with short dsRNAs of random base sequences were investigated, with a view to identify the nature and essential elements of their interactions.

\section{MATERIALS AND METHODS}

Nucleic acids used in this study include a small-interfering RNA labelled with 6-carboxyfluorescein (FAM-labelled dsRNA), 27 base pair (bp) RNA/DNA 1 and 2 formed from randomly selected complementary base sequences $\left(5^{\prime}\right.$-cauucgcaugaugccagugguacuaac- $\left.3^{\prime}\right)$ and poly I:C. FAM-labelled dsRNA (siGLO transfection indicator small-interfering RNA) was purchased from GE Healthcare Life Sciences, Little Chalfont, UK (Dharmacon \#D-001630-0105). The rest was synthesized by Sigma Aldrich (Dorset, UK). In all, 27 bp RNA/DNA 1 and 2 were reconstituted to $100 \mu \mathrm{m}$ stock solution in $1 \times$ phosphate-buffered saline (PBS), and aliquots stored at $-20^{\circ} \mathrm{C}$. To obtain the unlabelled 27-bp dsRNA and double-stranded DNA (dsDNA), equal volumes of the complementary single-strand stock solutions were mixed, then heated to $65{ }^{\circ} \mathrm{C}$ for $5 \mathrm{~min}$ to denature any secondary structures formed during storage, and allowed to cool slowly to enhance efficient annealing. Aliquots of the 27-bp dsRNA and dsDNA were then stored at $-20^{\circ} \mathrm{C}$. Radio-labelled $27 \mathrm{bp}$ dsRNA was obtained by labelling one strand of the 27-bp RNA with ${ }^{32} \mathrm{P}$ (to a final concentration of $100 \mathrm{~nm}$ ) using Adenosine 5 '-triphosphate $\left[\gamma^{-32} \mathrm{P}\right]$ (PerkinElmer, London, UK) and T4 Polynucleotide Kinase (Thermo Scientific, Fermentas, Loughborough, UK) according to the manufacturer's instructions. The radioactive strand was then washed with the QIAquick nucleotide removal kit (Qiagen \#28304, Manchester, UK) and kept at $4{ }^{\circ} \mathrm{C}$. It was subsequently annealed with the complementary strand just before use. Minocycline, doxycycline and magnesium sulfate were also purchased from Sigma ${ }^{\infty}$ Aldrich. Adenosine 5 '-triphosphate $\left[\gamma^{3}{ }^{32} \mathrm{P}\right]$ for radioactive labelling was obtained and T4 Polynucleotide Kinase. Nucleotide removal was done with QIAquick nucleotide removal kit (Qiagen \#28304). All buffers and reagent solutions for experiments involving RNA were reconstituted in diethylpyrocarbonate-treated water to inactivate RNases.

\section{Gel electrophoresis binding studies}

The interaction of minocycline and doxycycline with dsRNA was studied by gel electrophoresis using either $1 \%$ agarose gels in $1 \times$ TBE or $10 \%$ native polyacrylamide gel. ${ }^{24,25}$ The effect of this interaction on dsRNA band intensity was initially investigated with $200 \mathrm{~nm}$ FAM-labelled dsRNA in $1 \times$ NEBuffer 2 (New England Biolabs, Hitchin, UK), which contains $50 \mathrm{~mm} \mathrm{NaCl}, 10 \mathrm{~mm}$ Tris$\mathrm{HCl}, 10 \mathrm{~mm} \mathrm{MgCl}_{2}, 1 \mathrm{~mm}$ Dithiothreitol, $\mathrm{pH} 7.9$ at $25^{\circ} \mathrm{C}$. After $\mathrm{Mg}^{2+}$ was identified as the critical component of the buffer for an observable effect on band intensity, subsequent experiments were performed with unlabelled random $27 \mathrm{bp}$ dsRNA $(500 \mathrm{~nm})$ in $0.5 \times \mathrm{PBS}+5 \mathrm{~mm} \mathrm{MgSO}_{4}$. For these experiments, a range of concentrations of minocycline or doxycycline $(0-$ $1000 \mu \mathrm{M})$ were added to $1.5 \mathrm{ml}$ microcentrifuge tubes containing the dsRNA in buffer. The mixtures were incubated for 15-30 $\mathrm{min}$ at room temperature before loading in the gel. Binding competition assays were done by adding $1 \times$ ethidium bromide $(\mathrm{EtBr})\left(0.5 \mu \mathrm{g} \mathrm{m}{ }^{-1}, \approx 1.27 \mu \mathrm{M}\right)$ into the sample either before or after adding minocycline/doxycycline. Electrophoresis was performed at $5 \mathrm{v} \mathrm{cm}^{-1}$ in $0.5 \times$ TBE $(30 \mathrm{~V}$ for $90 \mathrm{~min}$ using Bio-Rad PowerPac and electrophoresis apparatus). For gel mobility shift assays, ${ }^{26}$ electrophoresis was done using fine resolution gel ( $10 \%$ native polyacrylamide gels) that would detect small differences in migration undetectable with agarose gels, and running the gel for longer $(3-4 \mathrm{~h}$ at $150 \mathrm{~V})$ to ensure adequate separation of the bands. When unlabelled dsRNA was used in the samples, gels were stained with $\mathrm{EtBr}$ (unless otherwise stated) by soaking the gels in $1 \times \mathrm{EtBr}$ solution $\left(0.5 \mu \mathrm{g} \mathrm{ml}^{-1}\right)$ for $15-20 \mathrm{~min}$ after electrophoresis and destained in distilled water for $10 \mathrm{~min}$. Images were captured using G:Box camera (SynGene, Cambridge, UK) and GeneSnap image software. For experiments involving radio-labelled dsRNA, the concentration of minocycline or doxycycline and
dsRNA used were proportionately reduced from the concentrations used for the FAM-labelled and EtBr-stained experiments to acceptable levels for working safely with radioactivity ( $5 \mathrm{~nm}$ dsRNA and $0-10 \mu \mathrm{m}$ minocycline/doxycycline). Samples were incubated at $37^{\circ} \mathrm{C}$ for $30 \mathrm{~min}$ before loading in wells of $10 \%$ native acrylamide gel. Gels were dried in a gel dryer after electrophoresis and exposed in a film cassette overnight. Images were taken with a Fujifilm image reader (FLA-3000 series v1.8, Bedford, UK) and analyzed using the Fujifilm Multiguage v3.0 image software (Bedford, UK).

\section{Fluorescence spectroscopy}

The changes in the absorbance and fluorescence emission spectra of minocycline/doxycycline upon binding to RNA were studied by fluorescence spectroscopy. ${ }^{27-29}$ A range of concentrations of both dsRNA $(0-20 \mu \mathrm{M})$ and minocycline/doxycycline $(0-500 \mu \mathrm{M})$ in Tris buffer $(\mathrm{pH} 8)+5 \mathrm{~mm} \mathrm{MgSO}_{4}$ were used in a 96-well plate. The absorbance and fluorescence emission spectra of the samples were monitored using a SpectraMax M2 microplate reader operated with the SOFTmax PRO data collection software (Molecular Devices, Wokingham, UK). The optimal excitation wavelength of minocycline/doxycycline was first determined by measuring the absorbance spectra. The fluorescence emission spectra were subsequently monitored with the excitation wavelength fixed at the pre-determined maximum absorption wavelength.

\section{Circular dichroism}

The binding of the tetracyclines to dsRNA was also studied by CD as described by Khan and Musarrat ${ }^{28,30}$ using poly I:C titrated against a range of concentrations of minocycline/doxycycline $(0-50 \mu \mathrm{M})$. The CD spectra of a fixed concentration of poly I:C $(50 \mu \mathrm{M})$ were first obtained, as well as that of the highest concentrations of minocycline and doxycycline used. The fixed concentration of poly I:C was then titrated against increasing concentrations of the drugs, and the CD spectra of the dsRNA upon interaction with the given concentrations of minocycline or doxycycline were recorded.

\section{RNase protection assay}

The effect of doxycycline on RNase III cleavage and consequent degradation of dsRNA was studied by using ShortCut RNase III (New England Biolabs) to digest 27 bp dsRNA in the absence and presence of $1 \mathrm{~mm}$ doxycycline following the manufacturer's protocol. Samples were collected from the reaction mixture at given time points. Upon sample collection, the reaction was stopped by transferring collected samples to tubes containing $5 \times$ EDTA on ice before loading in $1 \%$ agarose gels. Electrophoresis was carried out at $30 \mathrm{~V}$ for $120 \mathrm{~min}$, and the gels were stained with EtBr. Images were taken with SynGene G:Box camera using the GeneSnap image software.

\section{Data analysis}

RNA bands were quantified using the image processing software, GeneTools from SynGene (Cambridge, UK). Statistical analysis (multiple regression) was done using SPSS Statistics version 19 (IBM, London, UK).

\section{RESULTS}

\section{Effects of the tetracyclines on fluorescence intensity of dsRNA} bands

Agarose gel electrophoresis of FAM-labelled dsRNA to which a range of concentrations of minocycline or doxycycline had been added showed a decrease in the fluorescence intensity of the dsRNA bands with increasing amounts of minocycline or doxycycline (Figure 1a). This indicates some kind of interaction between the tetracyclines and dsRNA. To investigate the nature of these interactions, the experiments were repeated using the non-labelled 27-bp dsRNA of random sequence, and the gel was stained with $\mathrm{EtBr}$ after electrophoresis. Again, there was decreasing band intensity with increasing concentration of the tetracyclines (Figure 1b). The observed decrease in band intensity of dsRNA with increasing concentrations of the tetracyclines appears similar to the band intensity of lower amounts of dsRNA in the absence of the tetracyclines (Figure 1c). This 
a

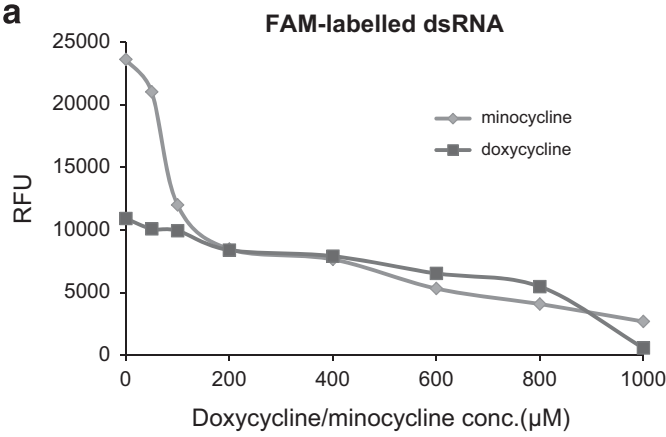

C

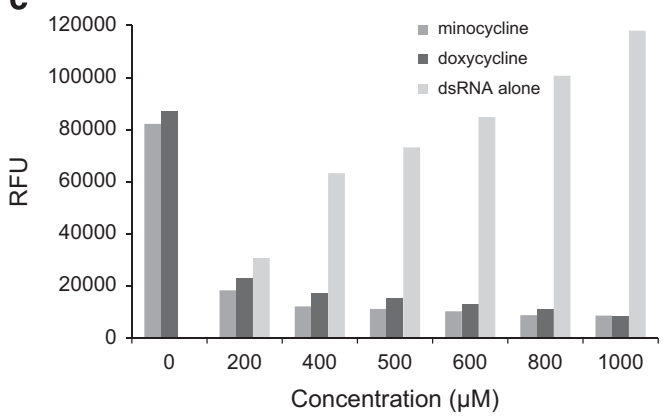

b

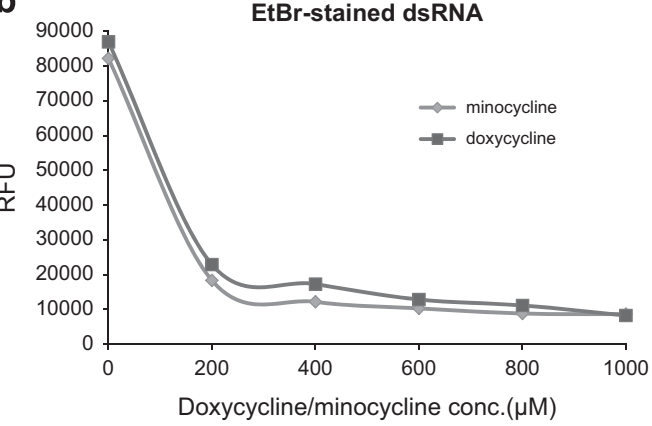

d

Tet. Conc $(\mu \mathrm{M})$

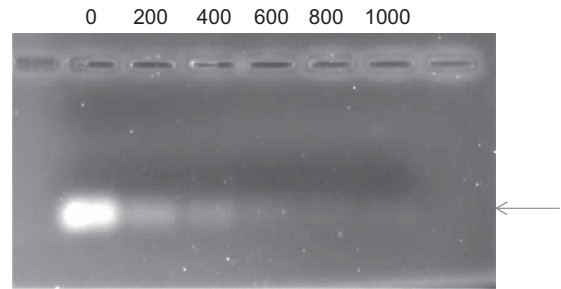

Figure 1 Effect of the tetracyclines (minocycline and doxycycline) on double-stranded RNA (dsRNA) band intensity. Graphs show decreasing dsRNA band intensity of (a) $200 \mathrm{~nm}$ small-interfering RNA (siRNA) (6-carboxyfluorescein (FAM)-labelled dsRNA); (b) 500 nm unlabelled 27 bp dsRNA (stained with ethidium bromide (EtBr) after electrophoresis) with increasing concentrations of minocycline or doxycycline. (c) Band intensities of various concentrations (0-1000 nm) of EtBr-stained $27 \mathrm{bp}$ dsRNA (without tetracycline) compared with $500 \mathrm{~nm}$ dsRNA in increasing concentrations of minocycline/doxycycline. (d) A sample gel image (of b). Arrow indicates dsRNA bands. RFU, relative fluorescence unit. A full color version of this figure is available at The Journal of Antibiotics journal online.

suggests that the interaction of the tetracyclines with dsRNA effectively reduces the amounts of dsRNA (binding sites) available for $\mathrm{EtBr}$ binding in the samples during staining, even though the same amount of dsRNA was used in these samples.

The effects of $\mathrm{Mg}^{2+}$ on the binding of the tetracyclines to dsRNA When the previous experiments involving FAM-labelled dsRNA were conducted with water or PBS in place of NEBuffer 2, there was no observable effect of tetracycline on the band intensity. Hence, the component of the buffer essential for the observed effect on band intensity was investigated. When the experiments were conducted in PBS buffer in the absence or presence of increasing concentrations of $\mathrm{Mg}^{2+}$, the gel showed decreasing dsRNA band intensity with increasing concentration of minocycline or doxycycline in the samples that contain $\mathrm{Mg}^{2+}$. This decrease in dsRNA band intensity was obscure in the absence of $\mathrm{Mg}^{2+}$, and was enhanced with increasing concentrations of $\mathrm{Mg}^{2+}$ (Figure 2). Similar results were also obtained for minocycline. These results indicate that $\mathrm{Mg}^{2+}$ enhances the interaction of the tetracyclines with dsRNA. In the samples containing doxycycline, a diffuse fluorescence increasing in a dose-dependent manner was observed above the dsRNA bands at higher drug concentration. This was not observed in the samples containing minocycline. This diffuse fluorescence was subsequently found to be coming from free doxycycline, as it was not observed when the samples were run without the antibiotics, and became more diffuse and widespread in antibiotic samples run without dsRNA. This therefore suggests that the tetracyclines may interact with dsRNA by binding, leaving the unbound drug molecules or molecules that dissociate from dsRNA to fluoresce above the RNA bands.

\section{Effects of tetracycline on dsRNA migration (electrophoretic} mobility shift assay)

The observed decrease in dsRNA band intensity in the presence of increasing amounts of the tetracyclines could result from precipitation of the RNA or differential migration of bound and unbound dsRNA. This would give rise to smearing of the RNA bands instead of a single/sharp band that would ordinarily be seen. To eliminate this possibility, gel mobility shift assay was done. The results showed no difference in the migration level of the bands from the top of the wells, and no accumulation of precipitates on the top of the wells (Figure 3a) in the samples containing both minocycline and doxycycline. These results suggest that the decrease in band intensity of dsRNA observed with the tetracyclines is not because of changes in the mobility of the complex formed by the interaction through the gel during electrophoresis. On the other hand, doxycycline was found to accumulate on the top of the wells at high drug concentrations. Again, this accumulation of drug molecules on the top of the wells was not observed for minocycline. This further indicates that doxycycline binds to dsRNA and migrates alongside dsRNA through the gel during electrophoresis, leaving only the unbound drug molecules at higher concentrations to accumulate in the wells.

To further test the possibility of the tetracyclines binding to dsRNA and keep track of the RNA molecules while avoiding the complications of interference from drug fluorescence, radioactively-labelled RNA was used. The result showed that the primary dsRNA band intensity remained fairly consistent (increased slightly with increasing drug concentrations), indicating that the dsRNA is not precipitated out of the sample during electrophoresis (Figure 3b). This suggests that the earlier observed decrease in band intensity of FAM-labelled and EtBr-stained dsRNA in the 

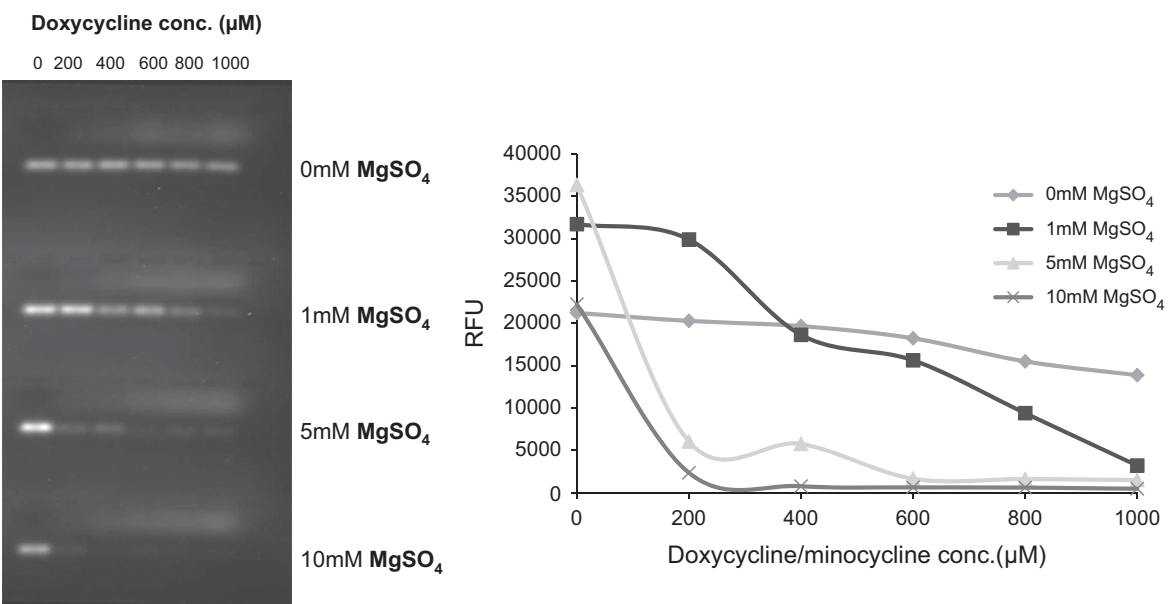

Figure 2 Effect of $\mathrm{Mg}^{2+}$ on the interaction of the tetracyclines with double-stranded RNA (dsRNA). Gel image and graphs show decreasing dsRNA band intensity of $200 \mu \mathrm{m}$ 6-carboxyfluorescein (FAM)-labelled dsRNA with increasing concentrations of doxycycline, which is enhanced in the presence of increasing concentrations of $\mathrm{Mg}^{2+}$. Note that the diffuse fluorescence increasing from left to right above the dsRNA bands in the gel image is from free doxycycline. A full color version of this figure is available at The Journal of Antibiotics journal online.

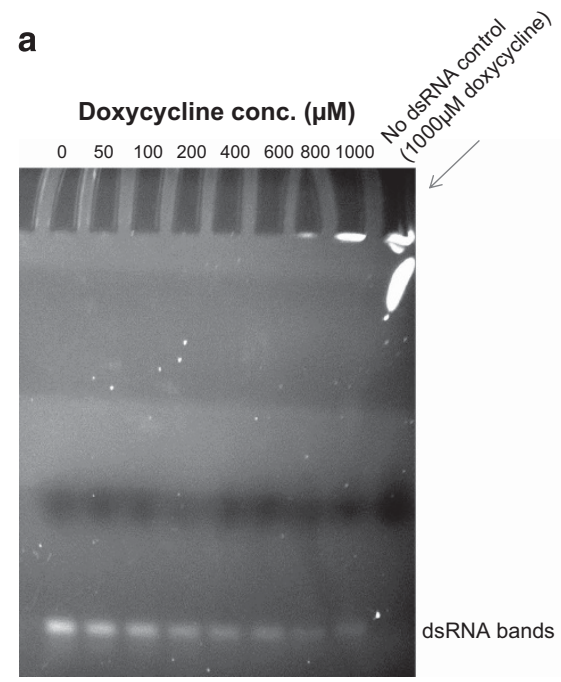

b

RADIO-LABELLED dSRNA
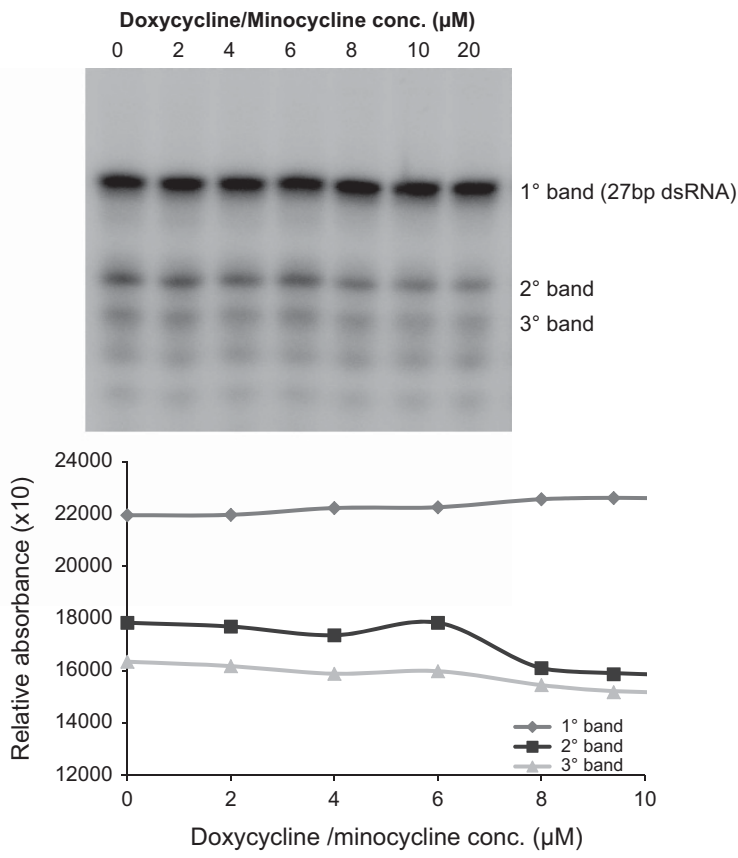

Figure 3 Effect of the tetracyclines on double-stranded RNA (dsRNA) migration through a high resolution gel (10\% native polyacrylamide gel). (a) Ethidium bromide (EtBr)-stained gel image shows no difference in gel shift/mobility of the dsRNA bands in the presence of increasing doxycycline concentrations, no smearing of the bands and no accumulation of dsRNA precipitates in the wells. Note the accumulation of free doxycycline on the top of the last two wells with high drug concentration. Arrow indicates lane of sample containing the highest concentration of doxycycline used without dsRNA. (b) Gel and graph of relative absorbance of radioactively-labelled dsRNA in the presence of increasing concentrations of doxycycline or minocycline. Note that the secondary and tertiary bands are degradation products of the dsRNA in the primary band. A full color version of this figure is available at The Journal of Antibiotics journal online.

presence of increasing concentrations of the tetracyclines is not due to loss of RNA during electrophoresis. However, there was a marked decrease in the intensity of the smaller secondary and tertiary bands (which are degradation fragments of the radioactivelylabelled $27 \mathrm{bp}$ dsRNA). This suggests that the binding of the tetracyclines to dsRNA may protect dsRNA from radioactivity-induced degradation. The slight increase in band intensity with increasing drug concentration appears to suggest a slightly higher amount of dsRNA. However, given that the same amount of dsRNA was used in all the samples, the secondary and tertiary bands with higher intensity at lower drug concentrations may account for this apparent difference. 
Tetracyclines inhibits binding of nucleic acid ligands to dsRNA If tetracycline binds to dsRNA, then it would be expected to compete with the binding of other molecules that are known to bind dsRNA. The effect of the interaction of the tetracyclines with dsRNA on the binding of a known nucleic acid ligand (EtBr) and the relative strength/affinity of the binding of the tetracyclines to dsRNA were investigated by adding $1 \times \operatorname{EtBr}\left(0.5 \mu \mathrm{g} \mathrm{ml}^{-1}\right)$ to the sample mixture before or after the addition of the tetracyclines. The result showed that the presence of the tetracyclines led to a dosedependent decrease in dsRNA band intensity, irrespective of which of the two compounds was first added to the sample (Figures $4 \mathrm{a}$ and $\mathrm{b}$ ). These results indicate that the tetracyclines reduce the binding of EtBr to dsRNA, and may be able to displace EtBr bound to dsRNA. Although these experiments were done with high tetracycline-EtBr molar ratios in the samples (for safety concerns), the gels were further soaked in EtBr solution to ensure maximum detection of dsRNA bands. After staining with EtBr, the gels still showed a dose-dependent decrease in band intensity with the tetracyclines, suggesting that $\mathrm{EtBr}$ is not able to completely displace bound tetracyclines from dsRNA.
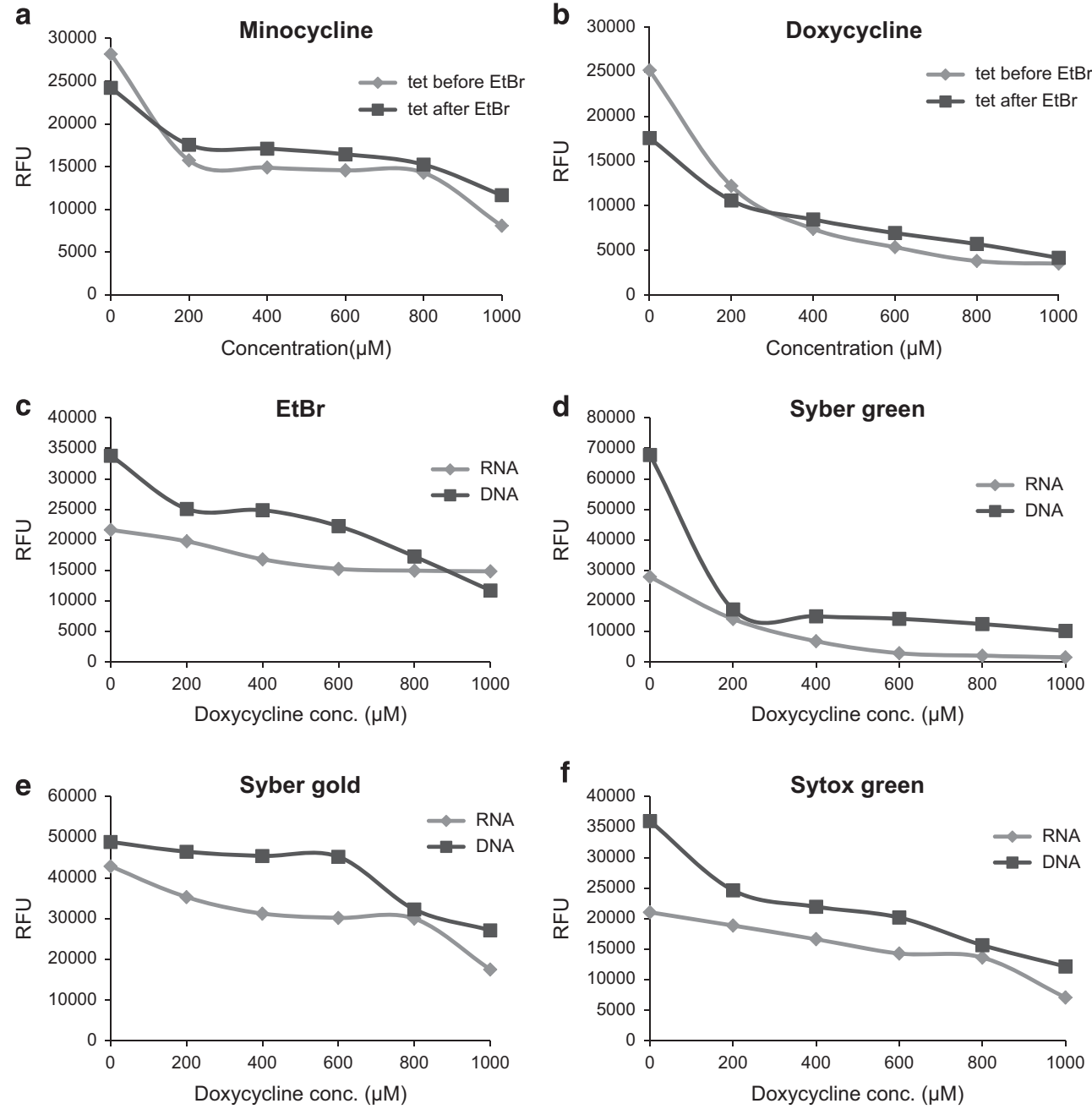

Figure 4 Effect of the tetracyclines on the binding of nucleic acid ligands to double-stranded RNA (dsRNA) and double-stranded DNA (dsDNA). (a, b) Competition assay graphs show decreasing band intensities of $500 \mu \mathrm{m} 27 \mathrm{bp}$ dsRNA with increasing minocycline/doxycycline concentration in the presence of $1 \times$ ethidium bromide (EtBr), irrespective of which compound was added first to the dsRNA (the tetracyclines or EtBr); decreasing band intensities of dsRNA and dsDNA with increasing concentrations of doxycycline in gels stained with EtBr (c), SYBR green (d), SYBR gold (e) and SYTOX green (f). A full color version of this figure is available at The Journal of Antibiotics journal online. 
absorption and emission wavelengths (not shown). Therefore, these experiments were done in Tris buffer $(\mathrm{pH} \mathrm{8})$, with $5 \mathrm{~mm} \mathrm{MgSO}_{4}$. In these experimental conditions, the maximum absorption wavelength of doxycycline was found to be $370-375 \mathrm{~nm}$, while that of minocycline was $380-385 \mathrm{~nm}$ ( $350 \mathrm{~nm}$ for both without $\mathrm{Mg}^{2+}$ in the buffer). The absorbance peak of both drugs was found to increase slightly (about $8 \%$ for doxycycline and 3\% for minocycline) upon the addition of RNA, although a clear dose-response effect was not observed.

The wavelength at which the maximum fluorescence emission was observed for doxycycline and minocycline was $515 \mathrm{~nm}$ and $410-415 \mathrm{~nm}$, respectively. The emission peak of doxycycline was found to decrease with increasing concentrations of dsRNA. On the other hand, the emission peak of minocycline was found to increase with increasing concentrations of dsRNA (Figure 5a). These changes are indicative of a binding interaction, and the extent of increase or decrease may be suggestive of the binding modes (depending on the dye/base pair ratio (dbpr)). These results therefore suggest that both minocycline and doxycycline bind to dsRNA, but may do so via slightly different modes such as different degrees of intercalation and surface/groove binding.

\section{Effects of the interaction with tetracyclines on the CD spectra of} dsRNA

The effect of the interaction of the tetracyclines with dsRNA on the dsRNA structure and conformation was assessed by monitoring the CD spectra of a given concentration of dsRNA (poly I:C) in the absence and presence of increasing concentrations of minocycline and doxycycline. Double-stranded poly I:C $(50 \mu \mathrm{M})$ showed a characteristic
CD spectrum with two positive bands at 240 and $278 \mathrm{~nm}$, respectively, and no negative band. The interaction of both drugs with dsRNA led to an increase in the positive band wavelength (red shift). With increasing amounts of minocycline $(0-50 \mu \mathrm{M})$, there was an increase in the ellipticity of both positive bands until saturation at $45-50 \mu \mathrm{M}$ minocycline, with a shift in the wavelength of the bands to 245 and $295 \mathrm{~nm}$, respectively (Figure 5b). Concomitant with these changes in the intrinsic CD of the dsRNA, two negative bands that also increased in ellipticity with increasing amounts of minocycline were observed at 265 and $340 \mathrm{~nm}$, respectively. When titrated with doxycycline, the positive band at $240 \mathrm{~nm}$ was no longer observed and there was a shift in the second positive band from 278 to $290 \mathrm{~nm}$ (Figure 5b). The ellipticity of this positive band also increased with increasing amounts of doxycycline (up to a maximum at $40 \mu \mathrm{M}$, then decreased). However, the maximum band intensity was still lower than the intrinsic dsRNA band. In addition, a negative band with similar characteristic was also observed at $265 \mathrm{~nm}$. These changes in the CD spectra of the dsRNA molecules upon interaction with minocycline or doxycycline are suggestive of structural alterations of the dsRNA in complex with the drugs. These results therefore indicate that the tetracyclines bind to dsRNA to induce structural alterations. They also further indicate that there may be differences in the specific binding modes of minocycline and doxycycline respectively to dsRNA.

\section{Tetracycline inhibits RNase III activity in vitro}

In view of the results from the radioactively-labelled RNA (section 'Effects of tetracycline on dsRNA migration (electrophoretic mobility shift assay)'), the possibility that the binding of the tetracyclines to a

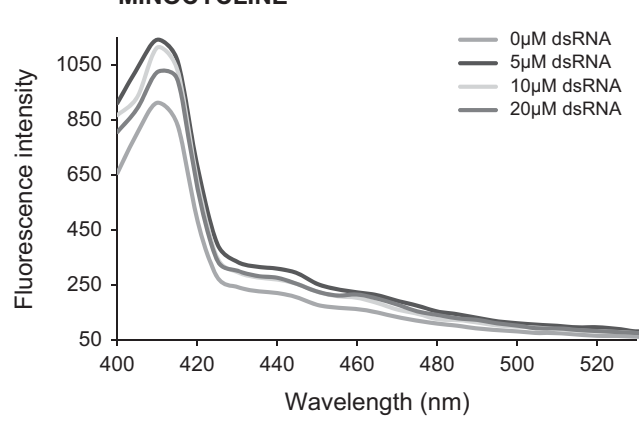

DOXYCYCLINE

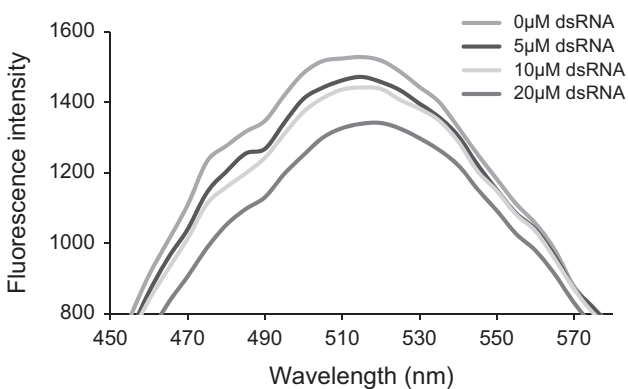

b
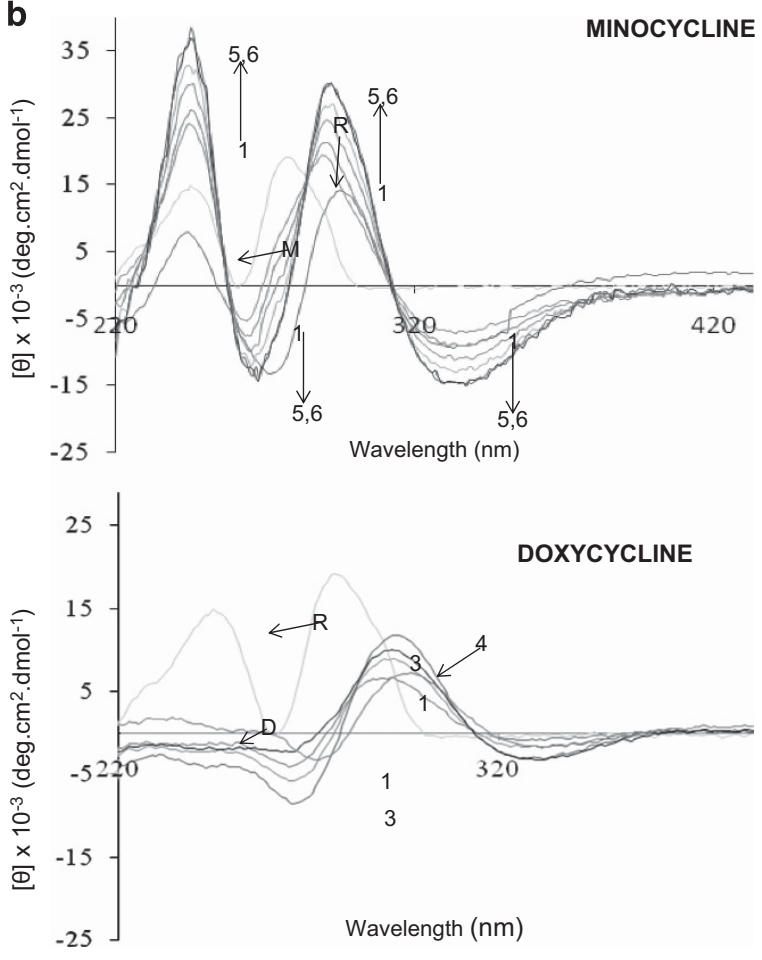

Figure 5 Effects of the interaction of the tetracyclines with double-stranded RNA (dsRNA) on the biophysical properties of the molecules. The graphs show the fluorescence emission spectra (a) of minocycline and doxycycline in the presence of increasing concentrations of 27 bp dsRNA (excitation wavelength was set at $375 \mathrm{~nm}$ ), and CD spectra of the dsRNA (poly I:C) in the presence of increasing concentrations of the drugs (b). For minocycline: 25, 30, 35, 40, 45, $50 \mu \mathrm{m}$ (curves 1-6); for doxycycline: 10, 20, 40, $50 \mu \mathrm{m}$ (curves 1-4). $\mathrm{M}$ and $\mathrm{D}$ represent the curves for minocycline and doxycycline, respectively, without any RNA, R represent CD spectra of $50 \mu \mathrm{m}$ poly I:C without any drug. A full color version of this figure is available at The Journal of Antibiotics journal online. 

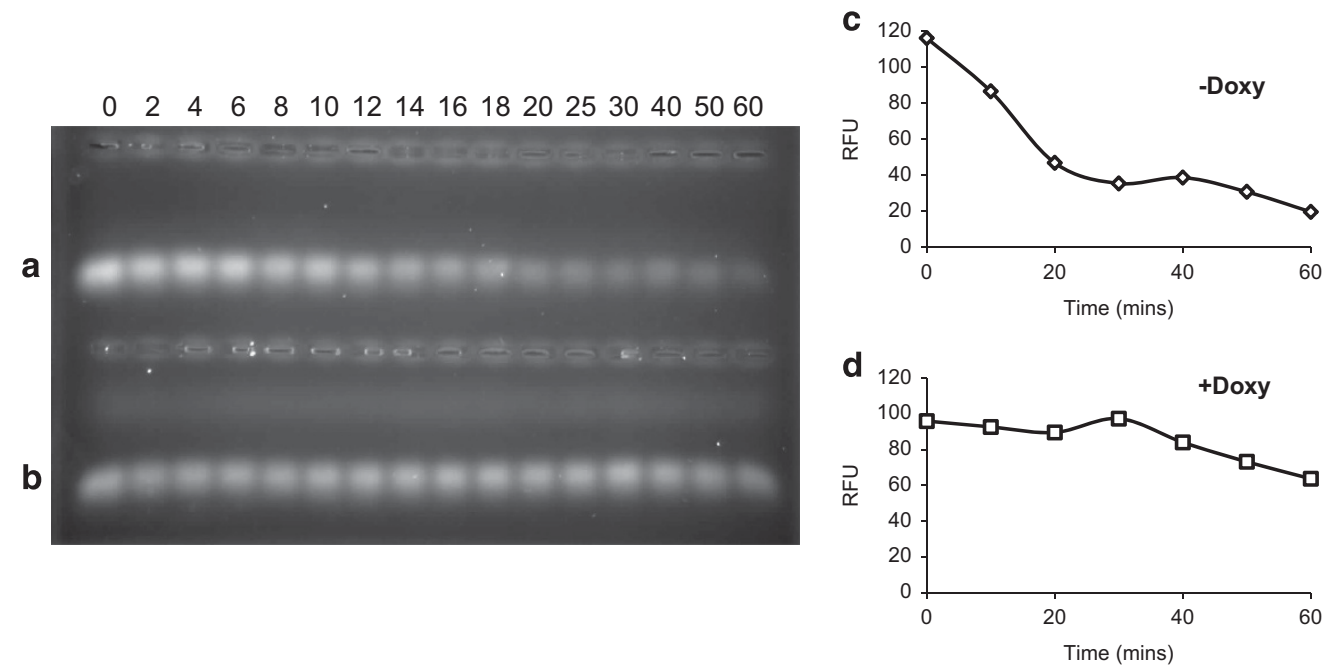

Figure 6 Effect of doxycycline on RNase III degradation of double-stranded RNA (dsRNA). Gel image shows (a) normal degradation of 27 bp dsRNA by RNase III (in the absence of tetracycline), (b) inhibition of degradation in the presence of $1 \mathrm{~mm}$ doxycycline. Samples were taken from the reaction mixture at the times (minutes) indicated above each well. The graphs show the rate of RNase III degradation of dsRNA in the absence (c) and presence (d) of doxycycline, with a significant difference between the two $\left(P=0.001\right.$ at 0.05 significance level, $\left.R^{2}=0.640\right)$. RFU, relative fluorescence unit of dsRNA bands.

dsRNA could offer some form of protection against degradation and/ or cleavage was assessed by measuring the rate of RNase III degradation of dsRNA in the absence and presence of increasing amounts of doxycycline. Degradation of the 27-bp dsRNA was observed as a decrease in fluorescence intensity of the band in gel electrophoresis, as it is too short to form distinct bands of RNase degradation products. Whereas RNA degradation began almost immediately in the sample without the drug and proceeded quite rapidly, onset of degradation was delayed in the sample containing doxycycline, and eventually occurred very slowly. Within the time studied in these experiments $(1 \mathrm{~h})$, RNase III degraded only about half of the dsRNA in the sample containing doxycycline, in contrast to a near complete degradation in the sample without the drug (Figure 6). Statistical analysis shows that doxycycline significantly reduced the rate of degradation of dsRNA (multiple regression analysis resulted in a $P$-value of 0.001 at 0.05 significance level, $R^{2}=0.640$ ). These results indicate that doxycycline inhibits dsRNA cleavage and degradation by RNase III, suggesting that doxycycline may interfere with RNase III processing/degradation of dsRNA. However, the eventual and slow degradation of dsRNA in the presence of doxycycline suggests that the binding of the drug to dsRNA may be reversible, and that the enzyme may eventually evade the protective effect of the drug.

\section{DISCUSSION}

Different base pairs have been indicated by several studies as the binding site of the tetracyclines to the $16 \mathrm{~S}$ ribosomal RNA. ${ }^{14-18}$ In view of the variations in these reports as to the exact target site(s), it is possible that the specific base pairs may not necessarily be the essential targets for the binding of the tetracyclines to rRNA. This study therefore investigated the binding of doxycycline and minocycline to short dsRNAs (seen in most cellular RNAs). The observed decrease in band intensity of the FAM-labelled dsRNA following electrophoresis in the presence of increasing amounts of the tetracyclines suggests an interaction with dsRNA. It is possible that the tetracyclines could quench FAM fluorescence when they interact with the FAM-labelled dsRNA. However, the observation of similar effect with unlabelled dsRNA stained with EtBr rules out this possibility. It is also possible that the binding of the tetracyclines to dsRNA could result in precipitation of the RNA, or a variable degree of mobility shift between bound and unbound dsRNA, as has been reported for some dsDNA ligands. ${ }^{26,31}$ This would give rise to smearing of the RNA bands instead of a single/sharp band that would ordinarily be seen. However, neither precipitation nor smearing of RNA bands was observed in this study.

$\mathrm{Mg}^{2+}$ was suspected to be the critical component of the reaction buffer for an observable effect, because it has been shown to enhance the cross-linking of bases in $30 \mathrm{~S}$ ribosomal subunit. ${ }^{32}$ The role of divalent metal ions in the binding of tetracyclines to DNA have been explored by Kohn, ${ }^{33}$ who noted that little or no tetracycline became bound to DNA in the absence of divalent metal ions, and magnesium was the most effective in enhancing the binding of tetracycline to the nucleic acid. There has also been speculation that the active drug species that binds to the ribosome is a magnesium-tetracycline complex. ${ }^{1,34}$ Therefore, these results concur that divalent cations like $\mathrm{Mg}^{2+}$ is an essential factor in the interaction of the tetracyclines with dsRNA, as the observed effects were enhanced in the presence of increasing concentrations of $\mathrm{Mg}^{2+}$.

$\mathrm{EtBr}$ is used as a stain for nucleic acids because its fluorescence increases several-fold upon binding (by intercalation) to nucleic acids, allowing detection of the nucleic acids by UV illumination. It then follows that when more $\mathrm{EtBr}$ is bound to a nucleic acid molecule, the band intensity will increase (up to a saturation point), and vice versa. Therefore, the simplest explanation for the tetracycline effect on dsRNA band intensity is that the number of dsRNA binding sites available for EtBr binding (used for staining) was reduced by the presence of tetracycline. In other words, the tetracyclines occupy the binding sites of $\mathrm{EtBr}$ in dsRNA, leaving fewer binding sites available for $\mathrm{EtBr}$ during staining. Hence, less EtBr is bound to the dsRNA in the presence of the tetracyclines, which is seen as lower band intensity for the same concentration of dsRNA. As EtBr is a well-characterized nucleic acid ligand, these observations therefore suggest that the tetracyclines also bind to dsRNA. Again, similar effects were observed with SYBR Green, SYBR Gold and SYTOX Green. A decrease in fluorescence intensity has been reported for the binding of SYBR green 
to dsDNA and total RNA at higher dbpr above the optimal, and this effect was found to be significantly enhanced by divalent cations. ${ }^{31}$ Since both the nucleic acid stains (dye) and dsRNA were used at the same concentrations in all the samples in this experiment, the decrease in fluorescence intensity observed with increasing tetracycline could therefore imply that the presence of tetracycline reduces the number of base pairs available for the stains to bind, thereby effectively increasing the dbpr. The results of the binding competition assay between doxycycline/minocycline and $\mathrm{EtBr}$ indicate that the tetracyclines not only compete with $\mathrm{EtBr}$ for their binding sites on dsRNA, but are able to displace EtBr from its binding sites in dsRNA. These results therefore suggest that the binding of the tetracyclines to dsRNA is relatively strong compared with that of EtBr, hence their ability to displace EtBr from the binding sites on dsRNA and the inability of EtBr to displace the tetracyclines from dsRNA. They also indicate that the tetracyclines bind to dsRNA in a way that may be similar to the binding of EtBr, SYBR Green, SYBR Gold and SYTOX Green to DNA; probably by intercalation and/or electrostatic surface binding, hence their ability to compete with these ligands for binding sites on the nucleic acids.

Tetracyclines are naturally fluorescent, and binding to larger molecules such as RNA alter their fluorescence properties. ${ }^{35}$ The fluorescence intensities of some fluorescent molecules are known to either increase or decrease upon binding to nucleic acids, depending on factors such as dbpr and the presence of cations, ${ }^{31,36,37}$ as was also observed in this study. It has been reported that the fluorescence intensity of tetracycline and doxycycline decreases upon binding to DNA, with doxycycline fluorescence being less quenched than that of tetracycline. ${ }^{28}$ In this study, however, the fluorescence intensity of minocycline was found to increase upon interaction with dsRNA while that of doxycycline decreased. This could be due to differences in the reactive functional groups of the drugs, which would invariably affect their binding characteristics. Although the fluorescence intensity of minocycline has been reported to be lower than that of doxycycline, ${ }^{35}$ the huge gap in the maximum emission wavelength of doxycycline and minocycline (515 and $415 \mathrm{~nm}$, respectively) observed in this study may account for the nearly non-existent fluorescence of minocycline recorded by Glette et al. ${ }^{35}$ as they only measured emission at $520 \mathrm{~nm}$.

The observed changes in CD spectra of dsRNA upon interaction with the tetracyclines indicate structural alterations due to binding of the drugs. An increase in ellipticity is a characteristic feature of the elongation of the duplex as a result of intercalation of the planar molecules between the stacked base pairs of nucleic acids and subsequent helix opening. ${ }^{28,38}$ Again, the differences in the nature and magnitude of the $\mathrm{CD}$ spectral changes on interaction with minocycline and doxycycline respectively indicate differences in their binding characteristics, probably due to differences in their reactive functional groups.

RNase III generally degrades dsRNAs both in vitro and in vivo. ${ }^{39}$ Inhibition of this process could have wide-ranging implications in living cells. In bacteria, for example, RNase III is essential for the initial processing of precursor rRNA transcripts for further maturation into the functional ribosomal RNA necessary for protein synthesis. ${ }^{40}$ In addition to the fact that the viruses against which the tetracyclines are effective are RNA viruses (West Nile fever virus, ${ }^{7}$ Japanese encephalitis virus $^{18}$ and human immunodeficiency virus $\left.{ }^{19-21}\right)$, most viruses produce dsRNA structures during replication. This could explicate the mechanism of their wide range of actions against various microbes.

\section{CONCLUSION}

This study showed that the tetracyclines (minocycline and doxycycline) bind to short dsRNAs of random base sequence and inhibit their cleavage by RNase III, indicating that the binding of the tetracyclines to RNAs may inhibit their processing, and consequently, function. As the functional forms of most cellular RNAs often involve secondary and tertiary structures formed by folding (leading to the formation of short double helices), it is possible that the binding of the tetracyclines to these double-stranded regions of various cellular RNAs may account for the wide range of therapeutic effects of the drugs.

\section{CONFLICT OF INTEREST}

The authors declare no conflict of interest.

\section{ACKNOWLEDGEMENTS}

This work was funded by the Commonwealth Scholarship Commission in the UK.

1 Chopra, I. \& Roberts, M. Tetracycline antibiotics: Mode of action, applications, molecular biology, and epidemiology of bacterial resistance. Microbiol. Mol. Biol. Rev. 65, 232-260 (2001).

2 Schnappinger, D. \& Hillen, W. Tetracyclines: antibiotic action, uptake, and resistance mechanisms. Arch. Microbiol. 165, 359-369 (1996).

3 Katiyar, S. K. \& Edlind, T. D. Enhanced antiparasitic activity of lipophilic tetracyclines role of uptake. Antimicrob. Agents Chemother. 35, 2198-2202 (1991).

4 Dutta, K. \& Basu, A. Use of minocycline in viral infections. Indian J. Med. Res. 133, 467-470 (2011).

5 Debrah, A. Y. et al. Doxycycline reduces plasma VEGF-C/sVEGFR-3 and improves pathology in lymphatic filariasis. PLoS Pathog. 2, e92 (2006).

6 Scheinfeld, N. \& Berk, T. A review of the diagnosis and treatment of rosacea. Postgrad. Med. 122, 139-143 (2010).

7 Michaelis, M., Kleinschmidt, M. C., Doerr, H. W. \& Cinatl, J. Minocycline inhibits West Nile virus replication and apoptosis in human neuronal cells. J. Antimicrob. Chemother. 60, 981-986 (2007).

8 Elewa, H. F., Hilali, H., Hess, D. C., Machado, L. S. \& Fagan, S. C. Minocycline for short-term neuroprotection. Pharmacotherapy 26, 515-521 (2006).

9 Ralph, S. A., D'Ombrain, M. C. \& McFadden, G. I. The apicoplast as an antimalarial drug target. Drug Resist. Updat. 4, 145-151 (2001).

10 Dahl, E. L. et al. Tetracyclines specifically target the apicoplast of the malaria parasite Plasmodium falciparum. Antimicrob. Agents Chemother. 50, 3124-3131 (2006)

11 Edlind, T. D. in Biochemical Protozoology (eds Coombs G. H. \& North M. J.) 569-586 (Taylor \& Francis Ltd: London, 1991).

12 Nonaka, L., Connell, S. R. \& Taylor, D. E. 16S rRNA mutations that confer tetracycline resistance in Helicobacter pylori decrease drug binding in Escherichia coli ribosomes. J. Bacteriol. 187, 3708-3712 (2005).

13 Oehler, R., Polacek, N., Steiner, G. \& Barta, A. Interaction of tetracycline with RNA: photoincorporation into ribosomal RNA of Escherichia coli. Nucleic Acids Res. 25, 1219-1224 (1997).

14 Pioletti, M. et al. Crystal structures of complexes of the small ribosomal subunit with tetracycline, edeine and IF3. EMBO J. 20, 1829-1839 (2001).

15 Brodersen, D. E. et al. The structural basis for the action of the antibiotics tetracycline, pactamycin, and hygromycin B on the 30 S ribosomal subunit. Cell 103 , 1143-1154 (2000).

16 Anokhina, M. M., Barta, A., Nierhaus, K. H., Spiridonova, V. A. \& Kopylov, A. M. Mapping of the second tetracycline binding site on the ribosomal small subunit of E.coli. Nucleic Acids Res. 32, 2594-2597 (2004).

17 Wei, Y. \& Bechhofer, D. H. Tetracycline induces stabilization of mRNA in Bacillus subtilis. J. Bacteriol. 184, 889-894 (2002).

18 Mishra, M. K. \& Basu, A. Minocycline neuroprotects, reduces microglial activation, inhibits caspase 3 induction, and viral replication following Japanese encephalitis. J. Neurochem. 105, 1582-1595 (2008).

19 Szeto, G. L. et al. Minocycline attenuates HIV infection and reactivation by suppressing cellular activation in human CD4+ T cells. J. Infect. Dis. 201, 1132-1140 (2010).

20 Zink, M. C. et al. Neuroprotective and anti-human immunodeficiency virus activity of minocycline. JAMA 293, 2003-2011 (2005)

21 Copeland, K. F. T., Brooks, J. I. \& Novel, A Use for an old drug: the potential for minocycline as anti-HIV adjuvant therapy. J. Infect. Dis. 201, 1115-1117 (2010).

22 Hermann, T. \& Tor, Y. RNA as a target for small-molecule therapeutics. Expert Opin. Ther. Pat. 15, 49-62 (2005).

23 Sucheck, S. J. \& Wong, C.-H. RNA as a target for small molecules. Curr. Opin. Chem. Biol. 4, 678-686 (2000).

24 Xavier, K. A., Eder, P. S. \& Giordano, T. RNA as a drug target: methods for biophysical characterization and screening. Trends Biotechnol. 18, 349-356 (2000). 
25 Lilley, D. M. J. in Methods in Enzymology vol 317: pp 368-393 (Academic Press, New York, NY, USA, 2000).

26 Furlan, R. L. A. et al. A rapid and sensitive method for the screening of DNA intercalating antibiotics. Biotechnol. Lett. 24, 1807-1813 (2002).

27 Walter, N. G. \& Burke, J. M. in Methods in Enzymology vol 317: pp 409-440 (Academic Press, New York, NY, USA, 2000).

28 Khan, M. A. \& Musarrat, J. Interactions of tetracycline and its derivatives with DNA in vitro in presence of metal ions. Int. J. Biol. Macromol. 33, 49-56 (2003).

29 Canzoneri, J. C. \& Oyelere, A. K. Interaction of anthracyclines with iron responsive element mRNAs. Nucleic Acids Res. 36, 6825-6834 (2008).

30 Sosnick, T. R., Fang, X. \& Shelton, V. M. in Methods in Enzymology vol 317: pp 393-409 (Academic Press, New York, NY, USA, 2000).

31 Zipper, H., Brunner, H., Bernhagen, J. \& Vitzthum, F. Investigations on DNA intercalation and surface binding by SYBR Green I, its structure determination and methodological implications. Nucleic Acids Res. 32, e103 (2004).

32 Noah, J. W., Dolan, M. A., Babin, P. \& Wollenzien, P. Effects of tetracycline and spectinomycin on the tertiary structure of ribosomal RNA in the Escherichia coli $30 \mathrm{~S}$ ribosomal subunit. J. Biol. Chem. 274, 16576-16581 (1999).

33 Kohn, K. W. Mediation of divalent metal ions in the binding of tetracycline to macromolecules. Nature 191, 1156-1158 (1961).
34 Chopra, I., Hawkey, P. M. \& Hinton, M. Tetracyclines, molecular and clinical aspects. J. Antimicrob. Chemother. 29, 245-277 (1992).

35 Glette, J., Sandberg, S., Hopen, G. \& Solberg, C. O. Influence of tetracyclines on human polymorphonuclear leukocyte function. Antimicrob. Agents Chemother. 25, 354-357 (1984).

36 Bhadra, K., Maiti, M. \& Kumar, G. S. Interaction of isoquinoline alkaloids with polymorphic DNA structures. Chem. Biodivers. 6, 1323-1342 (2009)

37 Yadav, R. C. et al. Berberine, a strong polyriboadenylic acid binding plant alkaloid: spectroscopic, viscometric, and thermodynamic study. Bioorg. Med. Chem. 13, 165-174 (2005)

38 Kumar, G. S., Das, S., Bhadra, K. \& Maiti, M. Protonated forms of poly[d(G-C)] and poly (dG).poly(dC) and their interaction with berberine. Bioorg. Med. Chem. 11, 4861-4870 (2003).

39 Srivastava, A. K. \& Schlessinger, D. Mechanism and regulation of bacterial ribosomal RNA processing. Annu. Rev. Microbiol. 44, 105-129 (1990).

40 Nikolaev, N., Schlessinger, D. \& Wellauer, P. K. 30 S pre-ribosomal RNA of Escherichia coli and products of cleavage by ribonuclease III: length and molecular weight. J. Mol. Biol. 86, 741-747 (1974). 